Mots. Les langages du politique

87 | 2008

Chrononymes. La politisation du temps

\title{
Ancien Régime. Pour une approche comparatiste du vocabulaire historiographique
}

Olivier Christin

\section{(2) OpenEdition \\ Journals}

Édition électronique

URL : https://journals.openedition.org/mots/11762

DOI : $10.4000 /$ mots. 11762

ISSN : 1960-6001

Éditeur

ENS Éditions

Édition imprimée

Date de publication : 21 juillet 2008

Pagination : 13-26

ISBN : 978-2-271-06685-5

ISSN : 0243-6450

\section{Référence électronique}

Olivier Christin, « Ancien Régime. Pour une approche comparatiste du vocabulaire historiographique », Mots. Les langages du politique [En ligne], 87 | 2008, mis en ligne le 30 mars 2011, consulté le 23 avril 2022. URL : http://journals.openedition.org/mots/11762 ; DOI : https://doi.org/10.4000/mots. 11762 


\section{Olivier Christin}

\section{Ancien Régime. \\ Pour une approche comparatiste du vocabulaire historiographique}

Dans un texte daté de 1988, justement considéré comme décisif dans l'histoire de la réflexion sur la Révolution et surtout sur la façon dont elle s'était elle-même pensée, François Furet avait longuement examiné les conditions historiques et politiques particulières à la fois de l'apparition de l'expression Ancien Régime autour de l'année 1789 et de sa fortune durable dans l'histoire politique de la France du $19^{\mathrm{e}}$ siècle ${ }^{1}$. Très loin de la notice fort sèche qu'un an auparavant le Dictionnaire de Jean Tulard avait consacrée à la même expression, l'analyse de François Furet se développait donc en deux temps.

\section{"Catch words as they rise " ${ }^{2}$}

Le premier était consacré à l'apparition de cette notion «consubstantielle à la Révolution française [et qui] en exprime l'envers, le mauvais côté, la négation». Sans délimiter de corpus particulier, comme les dictionnaires de la langue ou les discours politiques par exemple, Furet y rappelait, textes à l'appui, que l'expression circulait dès avant l'été 1789, par exemple dans certains cahiers de doléances, mais toujours dans un sens restreint et pour désigner un secteur particulier de l'administration et non la totalité du passé. Il montrait ainsi que ce n'est qu'avec la défaite des Monarchiens, la ruine de leurs espoirs d'accommoder souveraineté du peuple et autorité du Roi et l'abolition des privilèges, dans la fin de l'été 1789, qu'elle reçut véritablement sa pleine signification et, du même coup, sa pleine puissance politique et symbolique. L'échec du projet politique de ceux qui voulaient encore conserver aux prérogatives du Roi une place centrale dans le nouveau système politique et l'accélération du démantèlement des structures économiques et sociales, seigneuriales ou corporatives précipitèrent alors la cristallisation de

Université de Lyon, CNRS (LAHRA), olivier.christin@ens-lsh.fr

1. Furet, Ozouf, 1988 (je cite dans la réédition Flammarion, 1992, t. 4, p. 25-42).

2. William Dupré, 1801, Lexicographia-Neologia Gallica, Londres. 
l'expression Ancien Régime, qui en vint à désigner un mode d'organisation du monde social et une forme de gouvernement contraires aux droits de l'individu et à la liberté. Dans une lettre de 1790, Mirabeau pouvait ainsi écrire au Roi : "Comparez le nouvel état de choses avec l'Ancien Régime», et souligner par là la rupture avec le passé à laquelle il avait conscience d'assister3. En décembre 1790, le Dictionnaire national et anecdotique de Pierre-Nicolas Chantreau confirmait spectaculairement cette inflexion significative du lexique politique avec le passage de l'Ancien Régime corrompu et injuste au nouveau régime, en donnant cinquante exemples de transformation récente de mots comme citoyens ou aristocrates 4 ... Pour Furet, «en signant la fin de la tradition monarchique, l'écrasement des Monarchiens a inventé l'Ancien Régime ». La Révolution se construit dès lors dans l'assurance d'ériger $a b$ novo la société politique et de tout recommencer à partir d'une tabula rasa qui condamne définitivement le passé. La Constituante travaille dans la certitude d'avoir tout détruit de l'ordre ancien, de l'Ancien Régime, pour tout reconstruire, même si l'expression Nouveau Régime ne connait pas le même succès que son opposé.

L'invention discursive d'un passé constitué comme un tout et qu'il faut rejeter en bloc pour donner naissance à l'homme nouveau n'a pourtant rien d'évident et ce n'est que dans des circonstances très particulières que l'expression Ancien Régime peut s'imposer. Dans la langue prérévolutionnaire, régime revêt en effet une signification qui n'est qu'en partie liée à l'idée de gouvernement des hommes, de système politique, d'organisation du monde social. Certes, Jean Nicot en donne, au début du $17^{\mathrm{e}}$ siècle, une définition de ce type («Régime, ou gouvernement, Regimen, voyez Gouvernement, en Gouverner»5), mais les acceptions les plus courantes renvoient à l'administration d'un bien ou d'une communauté, à l'action de gouverner moralement quelqu'un ou une institution, par exemple à travers la règle d'un ordre religieux. En 1787-1788 encore, le Dictionnaire critique de Féraud ne comporte ainsi aucune entrée consacrée spécifiquement au «régime» comme gouvernement ou système politique et social, et il faut se reporter à l'entrée «régir » pour trouver une définition à la fois très brève et très lâche :

3. Cité par Tulard, Fayard et Fierro, 1987, p. 521.

4. Reichardt, 2005 [1801], col. 371-376. On doit comparer ce Dictionnaire national à celui, postérieur de quelques années, de William Dupré, Lexicographia-Neologia Gallica, Londres, 1801 - "The Neological French Dictionary containing the words of new creation not to be found in any french and english vocabulary hitherto published including those added to the Language by the Revolution and the Republic »-, mais celui-ci ne comporte aucune entrée spécifique à «Ancien Régime», dont on compte cependant plusieurs occurrences isolées - signe peut-être que, pour l'auteur, l'œuvre lexicale de la Révolution excède largement cette invention.

5. Jean Nicot, Thresor de la langue françoyse, 1606 ; j'utilise ici, comme pour les éditions successives du Dictionnaire de l'Académie française, l'édition en ligne proposée par http://www. atilf.fr. 
$1^{\circ}$. Gouverner. Il est bien difficile de bien régir un grand Peuple, un vaste Diocèse. $=$ En ce sens, il n'est que du style soutenu. $=2^{\circ}$. Au Palais, administrer. Régir une succession. Régir les Finances. $=3^{\circ}$. En Grammaire, exiger un certain régime.

Au cours de l'année 1789, une caricature célèbre de la monarchie absolue et de l'état d'infantilisation dans laquelle elle maintenait l'homme, intitulée Le Français d'autre-fois, illustre ainsi les incertitudes de la construction rhétorique du passé : elle montre un être joufflu ridicule, soutenu par un chariot en bois comme en ont les enfants qui ne savent pas marcher, tenant un hochet dans la main et assailli de toutes parts par les représentants de «l'ancienne police» : avocats, greffiers, procureurs, huissiers, secrétaires ${ }^{6}$... Le choix du terme police, pris ici dans son acception propre aux monarchies administratives de la période moderne, révèle les difficultés qu'il pouvait y avoir à trouver une expression capable de désigner précisément ce que l'éveil révolutionnaire condamnait à la disparition, car cette «police» parait bien n'être ici rien d'autre que l'administration elle-même et son armée de commis et d'officiers. L'évolution, autour de 1789, du traitement réservé au terme régime dans les dictionnaires et notamment dans les éditions successives du Dictionnaire de l'Académie française n'en est que plus révélatrice de l'effort lexical révolutionnaire. Alors que la quatrième édition (1762) se contentait d'expliquer que «RÉGIME en termes de Palais, signifie, Gouvernement, administration. Ainsi on dit, que Le Commissaire des saisies réelles est commis au régime \& administration des biens saisis, pour dire, qu'll est chargé de gouverner, d'administrer les biens saisis ", l'édition suivante, datée de 1798, ajoute à cette définition deux nouveaux paragraphes qui s'émancipent d'une acception étroitement administrative héritée des juristes des $16^{\mathrm{e}}-18^{\mathrm{e}}$ siècles pour faire du terme le moyen de désigner la nature du gouvernement et, au-delà, l'organisation même de la vie politique :

Il se dit De la manière de gouverner les États. Régime dur, arbitraire, absurde, inconstant, éclairé, bienfaisant. Le régime de ce Pays est despotique. Ce peuple est accoutumé à un régime doux.

Régime, signifie aussi, Administration, Gouvernement, dans un sens plus général. Ainsi on appelle Un nouveau Gouvernement, Le nouveau régime ; et L'ancien Gouvernement, L'ancien régime.

Le « régime » n'est donc plus seulement un mode d'administration, « une règle que l'on tient », un principe de gouvernement interne du Palais ou de certaines maisons religieuses : il désigne désormais aussi une forme de gouvernement de l’État et d'organisation de la société qui distingue de manière radicale deux

6. Caricature reproduite et commentée par de Baecque, 1988, p. 62-63. L'ancienne police est ici la borne de pierre qui empêche l'homme infantilisé de déplacer son chariot, de se rendre où il le souhaite et donc de conquérir sa liberté. 
moments de l'histoire. L'Ancien Régime est né et avec lui le Nouveau Régime, le nouvel «état de choses», la Révolution.

Par cette opération à la fois lexicale et politique, par ce double coup de force, la Révolution française se distinguait absolument, pour François Furet, des révolutions anglaises du $17^{\mathrm{e}}$ siècle, accomplies en bonne part au nom des anciennes libertés et des anciens droits, mais aussi de la révolution américaine qui a su ou pu faire l'économie d'une «liquidation» similaire du passé en faisant de l'Angleterre, et donc d'un pouvoir tenu pour étranger, l'incarnation de ce avec quoi elle entendait rompre. L'Amérique de la révolution n'a donc pas d'Ancien Régime et «cette absence est par excellence ce qui la constitue, son identité même». Furet soulignait ainsi la singularité de l'expérience révolutionnaire française qui, dans son aspiration à vouloir régénérer l'humanité par la liberté enfin arrachée à l'oppression, rejetait «le passé [qui] n'est plus qu'un Ancien Régime, définition lapidaire qui efface de la mémoire tout ce quil'a précédée».

L'invention de l'Ancien Régime aux fins des combats révolutionnaires euxmêmes portait pourtant avec elle des ambigüités ou des incertitudes inséparables de son efficacité et de son succès, que François Furet relevait. Celle, tout d'abord, de l'extension chronologique de l'Ancien Régime, dont la Révolution entendait faire table rase, en amont mais aussi en aval de 1789. Dès 1789 , en effet, de nombreuses lettres et feuilles volantes furent spontanément datées de l'An I de la liberté, comme la célèbre caricature, souvent copiée, J'savais ben qu'jaurions not tour7, inaugurant par là un calendrier révolutionnaire avant même que la Révolution en eût l'idée. En affirmant que la Révolution eut plusieurs «An I», scandés par l'été 1789, la fuite de Varennes et la proclamation de la République, et en rappelant que certains textes de 1792 distinguèrent un moment le temps écoulé depuis la conquête de la liberté (1789) et celui écoulé depuis l'établissement de l'égalité (1792), Furet soulignait les effets de la dynamique révolutionnaire elle-même, qui repoussait progressivement vers l'Ancien Régime ses premiers pas et ses premières expériences avant l'instauration de la République. La seconde difficulté tenait évidemment à la première et fit très vite, dans la perspective d'une œuvre révolutionnaire toujours à accomplir, sans cesse recommencée, de l'Ancien Régime non un passé révolu mais une menace présente, une virtualité politique, "un fantôme constant ». Sans doute est-ce d'ailleurs là l'une des raisons de l'échec de l'expression Nouveau Régime, qui ne pouvait désigner de manière satisfaisante ce qui était avant tout un processus, un mouvement appelé à se dépasser sans cesse lui-même. L'Ancien Régime devint le visage des multiples adversaires de la Révolution : les aristocrates et leurs complices, le clergé réfractaire, mais aussi la corruption des mœurs et les préjugés... Le couple des contraires

\section{Reproduite et commentée par de Baecque, 1988, p. 76-78.}


Ancien Régime / Révolution s'arrachait ainsi aux circonstances de son apparition pour revêtir un caractère abstrait et constituer par là même un héritage durable dans l'histoire politique de la France en servant à désigner deux «peuples» opposés dans leur façon de voir le passé.

C'est ici qu'intervenait le second temps, essentiel mais qu'il n'est pas ici nécessaire de reprendre, de la réflexion de François Furet, consacré aux usages politiques et savants de l'expression Ancien Régime dans la France du $19^{\text {e }}$ siècle et qu'un long chapitre des Lieux de mémoire allait, près de dix ans plus tard, compléter ${ }^{8}$.

\section{Concepts voyageurs}

En nouant histoire des conditions d'apparition et d'utilisation de l'expression Ancien Régime, d'une part, et histoire de sa fortune politique et scientifique au $19^{\text {e }}$ siècle, d'autre part, et en dévoilant par là les effets doublement structurants qu'eut cette invention lexicale et idéologique qui obligea les acteurs du $19^{\mathrm{e}}$ siècle à rejouer sans cesse les affrontements fondateurs de la Révolution, François Furet proposait une forme exemplaire de dénaturalisation de la langue politique et de réhistoricisation critique de ce qui est bien devenu «une évidence nationale, exposée et reçue comme allant de soi». Mais la comparaison même qu'il établissait avec les révolutions anglaises et américaines, son insistance sur le caractère «consubstantiel » à la Révolution française de la notion d'Ancien Régime et les quelques pages de l'analyse qu'il portait sur John Adams (dans lesquelles il affirmait : "Il existe un ancien régime anglais. Le mot n'existe pas chez John Adams, mais la chose, oui») suggéraient une autre opération possible d'objectivation critique de la langue et de ses fausses évidences dans le comparatisme. Peu explorée par Furet luimême - ce n'était d'ailleurs pas son propos -, cette perspective mérite d'être ici brièvement esquissée.

Si la formation Ancien Régime est consubstantielle à l'histoire même de la Révolution et strictement attachée à ce que François Furet nomme «circonstances », il faudrait, en toute rigueur, ne pas s'étonner de l'absence d'expressions comparables dans d'autres contextes politiques et dans d'autres circonstances, et tenir pour acquis son caractère intraduisible et singulier. Elle serait ainsi non un hapax, puisqu'elle se répand rapidement dans l'éloquence politique mais aussi dans la langue ordinaire dès 1789, mais une étiquette spécifique dont la production et la circulation s'avèreraient indissociables de la situation historique qui l'engendra et que par retour elle détermina durablement. À travers elle, la Révolution aurait établi sa singularité irréductible

8. Furet, 1997, p. 2301 et suiv. 
et en même temps son exemplarité. Les usages autochtones et les pratiques historiographiques récentes montrent pourtant qu'il n'en est rien et que les contemporains de la Révolution, ou plus exactement ceux qui en vécurent les retombées, comme les historiens, ne se sont pas satisfaits de cette singularité absolue. L'expression et la notion d'Ancien Régime leur ont très tôt paru avoir, hors même de la France de la fin du $18^{\text {e }}$ siècle, une pertinence théorique réelle 9 . Sans vouloir ici multiplier les exemples, on peut en prendre pour indice à la fois quelques citations de la littérature allemande du $19^{e}$ siècle, parfois antérieures à Tocqueville auquel Furet accordait une importance centrale dans la diffusion de l'expression, et la fréquence des titres qui reprennent le terme pour l'appliquer soit à des comparaisons entre la France et d'autres territoires, soit à des situations a priori différentes de celle de la France comme celle de la Suisse ${ }^{10}$, de l'Allemagne ${ }^{11}$ ou encore de l'Italie ${ }^{12}$.

Certes, certaines occurrences d'Ancien Régime dans la langue allemande $\mathrm{du} 19^{\mathrm{e}}$ siècle semblent soit trop allusives - comme lorsque Elise von Türckheim parle, dans une lettre de 1798 , de «régime », sans précision supplémentaire et sans référence explicite à l'invention lexicale de la Révolution -, soit étroitement liées aux évènements révolutionnaires et au passé qu'ils voulurent abolir, par exemple chez Wit von Dörring qui évoque en 1830 un «adhérant de l'Ancien Régime», ou chez Ludwig Bauer. D’autres usages, pourtant, révèlent une première extension des acceptions de l'expression, qui devient une manière de désigner non un régime particulier à la France, mais une époque révolue, un passé commun à l'Europe, un repère commode dans le temps : en

9. Voir, par Walter March [pseudonyme d'Orlando Bolivar Wilcox], Shoepac Recollections : a WaySide Glimpse of American Life, qui parle ainsi de sa ville : "At the time I came upon the stage of events, the transition to a modern American town had scarcely commenced. The body of the population was still of the Ancien Régime. » Voir aussi le choix de Thomas Hood (1799-1845) dans son New Dictionary of Quotations from the Greek, Latin, and Modern Languages - "Tr. into English, and occasionally accompanied with illustrations, historical, poetical, and anecdotal. With an extensive index, referring to every important word. By the author of "Live and learn", "The Newspaper and general reader's pocket companion", etc. From the last London ed.» - qui choisit de donner une entrée à Ancien Régime et de préciser que l'expression est d'origine française, mais ne réserve pas son usage à la seule situation de la France : «the former, old, government, old form of government, former administration », p. 30.

10. Hans-Rudolf Burri, 1975, Die Bevoelkerung Luzerns im 18. und fruehen 19. Jahrhundert: Demographie und Schichtung einer Schweizer Stadt im Ancien Régime, Lucerne, Rex-Verlag (Luzerner historische Veröffentlichungen Bd. 3) et Silvio Bucher, 1974, Bevölkerung und Wirtschaft des Amtes Entlebuch im 18. Jahrhundert : eine Regionalstudie als Beitrag zur Sozial- und Wirtschaftsgeschichte der Schweiz im Ancien Régime, Lucerne, Rex-Verlag (Luzerner historische Veröffentlichungen Bd. 1).

11. À titre d'exemple, on peut citer Ulrich-Christian Pallach, 1987, Materielle Kultur und Mentalitäten im 18. Jahrhundert: wirtschaftliche Entwicklung und politisch-sozialer Funktionswandel des Luxus in Frankreich und im Alten Reich am Ende des Ancien Régime, Munich, Oldenbourg, ou André Holenstein, 2003, "Gute Policey» und lokale Gesellschaft im Staat des Ancien Régime: das Fallbeispiel der Markgrafschaft Baden (-Durlach), Epfendorf, Bibliotheca Academica.

12. Parmi de nombreux titres, voir par exemple Marco Boscarelli, 1980, Dall'Ancien Régime a Maria Luigia in un centro minore degli Stati parmensi, Milan, Giuffré. 
1846, dans leurs échanges épistolaires, Theodore Fontane et Bernhard von Lepel emploient ainsi l'expression Ancien Régime, en français et de manière purement métaphorique, pour désigner un temps désormais lointain ${ }^{13}$. Certains dictionnaires contemporains semblent de même adopter une définition assez large, qui cesse de reposer sur la singularité de l'expérience française ${ }^{14}$.

Le plus important toutefois reste évidemment la fortune historiographique de l'expression, en dehors de la France, pour décrire et qualifier des situations historiques bien différentes de celles que Tocqueville avait à l'esprit lorsqu'il décrivait l'Ancien Régime, par exemple chez un Émile Lousse (La société d'Ancien Régime, 1956) ou un Ernest Neville Williams (The Ancien Régime Europe, 1970). En obligeant ceux qui s'y livraient à s'engager dans ce que la rhétorique désigne comme des applications, le comparatisme a ainsi constitué l'un des lieux essentiels de la critique des constructions chronologiques comme du lexique historique ${ }^{15}$, et c'est en bonne part une même opération qui invitait à s'interroger sur la pertinence d'une expression comme Ancien Régime et sur la réalité de la césure introduite par l'année 1789 dans l'histoire de nombre de pays européens. Pour comprendre à partir d'un corpus précis comment de telles applications sont possibles, sur quelle interprétation d'Ancien Régime elles se fondent et quels problèmes de périodisation de l'histoire elles soulèvent, il n'est peut-être pas inutile de se tourner vers quelques-uns de ces outils les plus courants et théoriquement les plus éloignés des usages politiques de la langue historique - que cela soit véritablement le cas ou non que sont les dictionnaires ou les lexiques historiques et observer le sort qu'ils réservent à l'expression prise au français.

Les Geschichtliche Grundbegriffe ${ }^{16}$, publiés à partir de 1972 parOtto Brunner, Werner Conze et Reinhart Koselleck, ne consacrent pas d'entrée spécifique à l'expression Ancien Régime, en français ou en traduction allemande. La période de référence que se donne cette immense entreprise de sémantique historique et certains de ses objectifs théoriques (décrire la disparition de l'ancien monde et l'émergence du nouveau dans leur expression conceptuelle, «la naissance langagière du nouveau monde et sa prise de conscience de soi [...] dans les concepts ${ }^{17}$ ) auraient pourtant pu laisser a priori supposer qu'une telle entrée aurait été légitime : il n’en est rien, parce que justement rien de comparable

13. Lettres d'Elise von Türckheim : Die Briefe derElise von Türckheim geb Schönemann: Goethes Lili, édité par J. Ries, 1924; Wit von Dörring, Fragmente aus meinem Leben und meiner Zeit, Leipzig, 1828-1830; Theodor Fontane, Bernhard von Lepel: Theodor Fontane und Bernhard von Lepel: ein Freundschafts-Briefwechsell, édité par J. Petersen, Munich, Beck, 1940; Ludwig Bauer, Die überschwänglischen, 1836. Références de ces citations dans Deutsches Fremdwörterbuch, Berlin, New York, De Gruyter, t. 3, 1977, entrée « régime».

14. Pierer's Universal Lexikon, 4 é édition, $1857-1865$, par exemple. Ancien Régime : « die Ruckkehr zur alten Staatsordnung, welche durch eine Revolution gestört war».

15. Bödeker, Hinrichs éd., 1991, notamment dans l'introduction.

16. Brunner, Conze, Koselleck éd., 1972-1992.

17. Introduction, Koselleck, p.xv. 
à Ancien Régime ne vient surgir dans la langue allemande de la Sattelzeit, de la période d'avènement de la modernité qui s'étire de 1750 à 1850 . Même constat d'absence pour le Dictionary of Eighteenth Century World History publié chez Blackwell par Jeremy Black et Roy Porter, qui s'ouvre même sur une critique des «appellations rebutantes» (off-putting) comme Ancien Régime avant de l'employer une page plus loin à propos d'un «Ancien Régime agraire ${ }^{18}$ et pour le Dictionnaire historique de la Suisse (désormais abrégé en DHS), trilingue.

Dans ce dernier, pourtant, l'expression connait un très grand nombre d'occurrences, dans les textes français, mais aussi dans les textes allemands ou italiens qui, généralement, ne la traduisent pas, et sur lesquels il faut revenir brièvement. Tout se passe en fait comme si le DHS hésitait sur le statut à donner à l'expression, choisissant ici le simple emprunt linguistique, là l'antonomase et la transformation d'une situation historique déterminée en phénomène général identifiable, avec des nuances, dans la Confédération. Dans le premier cas, les auteurs germanophones ou italophones du DHS emploient Ancien Régime pour désigner certains épisodes et certains acteurs précis des conflits qui se nouent en 1798 autour de l'intervention française et de la République helvétique : ainsi Frédéric-César de La Harpe, partisan précoce de la Révolution et de la République helvétique, membre du Directoire, «versuchte vergeblich, Frankreich klar zu machen, dass die enormen Kriegskontributionen die Schweiz auf die Seite der Anhänger des Ancien Régime trieb» ou «tentò invano di convincere la Francia che gli ingenti contributi di guerra richiesti spingevano gli Svizzeri nel campo dei fautori dell'ancien régime ${ }^{19}$. Le second cas est celui de la transformation du nom propre Ancien Régime en nom commun des sociétés préindustrielles : non sans incertitudes ou ambigüités, par exemple à propos de l'inclusion ou non de la fin du Moyen Âge, l'expression désigne explicitement l'époque antérieure à la République helvétique et donc à l'introduction des principes et des ambitions de la Révolution française dans l'ancienne Confédération. Ancien Régime renvoie ainsi à la fois à des formes d'organisation sociale, à des structures politiques, à des principes juridiques abolis ou suspendus par la révolution helvétique et ses protecteurs français. Mais cet usage même conduit à une extension progressive des significations de l'expression. À propos du colportage, par exemple, qui n'est, en toute rigueur, pas spécifique au système politique d'Ancien Régime, les auteurs du DHS précisent : "Während des ganzen Ancien Régime versuchten die Kantone und ihre zugewandten Orte vergeblich, die von den sesshaften Händlern als Konkurrenz empfundene Hausierereizu reglementieren», ou : «Durante tutto l'ancien régime, i cant. e i loro alleati tentarono invano di

18. Black, Porter éd., page xıv de l'introduction critique des expression rebutantes qui reviennent pourtant p. xv.

19. Le texte français donne : «ll a tenté en vain de montrer à la France que les énormes contributions de guerre exigées poussent les Suisses du côté des partisans de l’Ancien Régime. » 
regolamentare il commercio ambulante ${ }^{20}$. Les choix lexicaux du DHS - qui excluent une entrée spécifique mais font d'Ancien Régime une manière de désigner ce que la Révolution abolit, provisoirement ou définitivement - s'accordent ainsi en bonne part, non seulement avec l'extension progressive des acceptions qu'évoquait Furet, mais aussi avec la conviction d'une singularité de l'histoire de la Révolution française, puisque c'est bien son exportation vers la Confédération qui vient mettre un terme, en le nommant, à l'Ancien Régime suisse, pourtant différent de celui du royaume. Le DHS rejoint ici les frontières de l'Ancien Régime que Lucien Bély avait tracées en préambule à son Dictionnaire paru en 1996. Pour Bély, en effet, «l'Ancien Régime est une notion familière en France et dans la sphère d'influence de la France, là où justement la révolution a importé ses idées nouvelles et ses bouleversements » (p. xı). C'est donc en partie parce que la Révolution s'étendit au-delà des frontières que l'expression Ancien Régime peut être appliquée à des contrées où, certes, les «circonstances » qui préludèrent à son invention furent différentes, mais où l'on observe néanmoins les mêmes aspirations à faire table rase du passé.

Mais nombre d'ouvrages, aux ambitions d'ailleurs hétérogènes, renoncent à ces définitions strictes, qui font de l'invention lexicale des révolutionnaires un tournant décisif et le véritable acte de naissance de l'Ancien Régime et qui soumettent par là tout usage savant légitime de l'expression à la prise en compte exacte des conditions et des fonctions de son apparition. Dans l'abondante littérature des lexiques, dictionnaires et autres manuels - que la démocratisation de l'enseignement universitaire, mais aussi la volonté de réintroduire une forme de normativité lexicale dans une discipline en pleine évolution semblent avoir prodigieusement accrue - certains titres, notamment français, font le choix de ne rien définir, comme si l'évidence que relevait François Furet continuait de s'imposer21. Le Lexique historique de la France d'Ancien Régime (Cabourdin, Viard, 1998) ou le Dictionnaire de l'Ancien Régime dirigé par Robert Muchembled (2004) se passent ainsi de toute entrée propre pour Ancien Régime et, en fait, de toute définition et de toute allusion à l'origine de l'expression. Mais d'autres titres, français et étrangers, choisissent de tenter de définir l'Ancien Régime et, dans certains cas, d'en rappeler les conditions historiques et langagières de naissance.

Or ce choix les conduit à des positions presque opposées. Pour le Dictionnaire historique de Dominique Vallaud (1995), le Herder Lexikon²2, le Ploetz

20. Texte français: «Tout au long de l’Ancien Régime, les cantons et leurs alliés tentent, en vain, de réglementer le colportage...»

21. Voir les remarques de Reichardt (2005, col. 375) sur le caractère «évident» (selbstverständlich) du concept dans l'historiographie française.

22. Herder Lexikon. Geschichte 1 : Sachwörter mit über 2700 Stichwörter sowie über 300 Abbildungen und Tabellen, Fribourg, Bâle, Vienne, 1977. 
Geschichtslexikon 23 ou encore le Chambers Dictionary of World History24, l'Ancien Régime est «une organisation sociale et politique qui a prévalu en France depuis la fin de l'époque féodale jusqu'à la Révolution» (Vallaud), ou «une société et une organisation politique de l'Europe au temps de l'absolutisme $\left(17^{\mathrm{e}}-18^{\mathrm{e}}\right.$ siècle) notamment dans la France des Bourbons» (Herder), ou encore «le système social et politique qui a existé en France de la fin du XVIe siècle jusqu'à l'éclosion de la Révolution française» (Chambers). Ainsi réifié et détaché de ses conditions d'invention lexicale, l'Ancien Régime devient une réalité bien tangible et bien française qui correspond, avec plus ou moins de précision, à la monarchie des Bourbons. D'autres ouvrages, a priori destinés au même public élargi, s'enhardissent toutefois à proposer des définitions plus ambitieuses, mais aussi, du même coup, plus problématiques. Le Meyers kleines Lexikon (Forstmann, Hirschelmann, 1987), notamment, associe certes le terme à la société et au système politique français antérieur à la Révolution, mais il conclut cependant au caractère opératoire du concept hors des limites géographiques de la France : «Le concept peut être d'une manière générale utilisé pour caractériser l'état politique et social de l'Europe prérévolutionnaire ou ancienne Europe. Et ici, c'est essentiellement la révolution industrielle qui constituera la véritable rupture qui sépare la vieille Europe de la nouvelle. » Le Meyers, préfacé par Golo Mann, ignore ainsi la double restriction que proposait Lucien Bély : non seulement ce n'est pas la Révolution politique qui met fin à l'Ancien Régime, dans un moment de purvolontarisme politique prophétique, mais la césure brutale qu'il signifie déborde largement la zone d'influence de la France révolutionnaire. Ce choix n'est pas isolé : on le retrouve dans le classique Wörterbuch zur Geschichte de Fuchs et Raab25 et, dans une moindre mesure, dans l'Enciclopedia Storica sous la direction de Massimo Salvadori26. En proposant d'étendre la validité de la notion bien audelà de l'exemple français, ces dictionnaires entendent donc se livrer à une opération complexe qui l'émancipe largement des usages indigènes et donc du langage des acteurs historiques : peu importe ici qu'aucun contemporain n'ait parlé d'Ancien Régime pour décrire la situation économique et sociale qu'il évoquait, que le terme n'ait connu qu'une diffusion limitée. L'opération n'est pas forcément illégitime car, en faisant presque d'Ancien Régime un nom

23. Ploetz Geschichtslexikon. Weltgeschichte von A bis Z, Wurzbourg, 1986.

24. Chambers Dictionary of World History, Edimbourg, Chambers, 1993.

25. Konrad Fuchs, Heribert Raad, 1996, p. 43. Ancien Régime s’applique à la «situation avant la révolution de 1789; l'expression est souvent employée de manière dépréciative; synonyme d'ancienne Europe ».

26. L'ouvrage de Salvadori, 2000 , p. 53, retrace assez précisément les conditions d'apparition du terme dans l'année 1789, mais évoque aussi des acceptions plus larges (le terme «s'applique, par extension, à la période de l'histoire européenne qui est comprise entre le $x \mathbf{x}^{\mathrm{e}}$ et le XvıIIe siècle, durant laquelle se forment et se consolident les États absolutistes » et «le marxisme insère le concept d'Ancien Régime dans une théorie plus large du changement historique caractérisée [...] par la transition du féodalisme au capitalisme»). 
commun, elle rend compte de réalités historiques autrement inexprimables et qui purent d'ailleurs être inaperçues de ceux qui les vécurent (comme dans le cas de l'Ancien Régime démographique, par exemple). La relation étroite que François Furet établissait entre invention lexicale de la Révolution et langage de l'histoire est ici rompue.

Mais ces usages plus ou moins audacieux et surtout plus ou moins justifiés de l'expression Ancien Régime appellent plusieurs remarques. Alors qu'ils paraissent très dissemblables, ils s'accordent en fait profondément, comme le relève Rolf Reichardt, dans l'attribution à l'expression d'une connotation le plus souvent opposée à celle que lui donnait Tocqueville : «Dans la recherche moderne, Ancien Régime désigne les structures traditionnelles, quasi immobiles de l'ancienne Europe » et non, justement, un moment de transformation de ces structures par l'effort centralisateur de la monarchie. Ce retournement ne fait pourtant pas l'objet d'un commentaire ou d'une explication et les délicats problèmes de théorie de la modernité qu'il soulève restent au fond sans réponse, car se demander ce qu'est l'Ancien Régime ou quand il s'achève, c'est aussi s'interroger sur ce qui fait passer l'Europe d'un modèle de société à un autre. Du coup, aucun des choix lexicaux des dictionnaires destinés au public élargi des amateurs d'histoire et des étudiants n'est en mesure d'offrir de solution pleinement satisfaisante à la question de la périodisation et des conventions de chronologie, faute de porter au jour les présupposés méthodologiques et heuristiques sur lesquels ils reposent : une conception de la périodisation fortement dominée par l'histoire politique et militaire évènementielle chez ceux qui veulent réserver Ancien Régime à la France et à la diffusion progressive de l'influence révolutionnaire par les conquêtes militaires, et dans laquelle c'est bien la rupture politique inventée et désignée par la Révolution qui est facteur de changement historique; une référence implicite au temps long des sociétés européennes imaginé par Otto Brunner et Dietrich Gerhard chez ceux qui reprennent leur concept de Alteuropa et le donnent comme équivalent d'Ancien Régime, ignorant par là les différences idéologiques qui séparent les deux expressions ${ }^{27}$, et dans laquelle d'autres forces de changement, économiques ou démographiques par exemple, sont à l'œuvre.

Les quelques exemples convoqués ici - et que chacun peut aisément compléter tant la production éditoriale de lexiques, dictionnaires ou manuels proposant une nouvelle normativité lexicale sous couvert de neutralité axiologique semble enfler - suffisent sans doute à établir les conditions théoriques et pratiques qui doivent présider à l'analyse de certains chrononymes.

Ils invitent, en effet, à considérer ces derniers comme des productions idéologiques et scientifiques étroitement dépendantes de leurs conditions langagières de formulation et à y voir des formes d'évidence à la validité très

27. Sur ces questions, voir Bödeker et Hinrichs éd., 1991, notamment dans l'introduction. 
limitée, qui peinent à établir des points de repères communs aux différentes histoires et aux différentes historiographies : des "évidences nationales», pour reprendre l'expression de Furet, sans aucun doute, mais aussi parce qu'elles s'expriment dans des langues nationales. La storia contemporanea italienne ne recouvre donc pas l'histoire contemporaine française, pas plus que la Neuzeit allemande ou la modern history anglaise ne trouvent leur place naturelle dans l'époque moderne. Des précisions complémentaires peuvent, certes, être ajoutées, comme dans les expressions early modern ou Frühneuzeit, mais les hiatus entre les chrononymes et les découpages chronologiques nationaux ne sont pas pour autant abolis, car la question de savoir où la première modernité s'efface devant la modernité demeure et avec elle, justement, la question de la fin de l'Ancien Régime dans son propre acte de naissance langagier.

L'étude des chrononymes porte donc au jour des "évidences nationales» dont la force conceptuelle dépend et pâtit à la fois de la langue dans laquelle ils viennent à être formulés. Accomplir à leur sujet un véritable travail d’objectivation critique et de réflexivité historique peut donc exiger davantage que l'histoire de leurs conditions spécifiques d'apparition et que l'analyse de leur sédimentation progressive dans la mémoire nationale. Elle demande peutêtre un outil supplémentaire de mise à distance objectivante, de dénaturalisation de ce qui nous parait aller de soi, se passer d'explication (comme dans certains dictionnaires français), se comprendre à demi-mot entre collègues, de débanalisation du banal, que l'on peut espérer trouver dans le comparatisme et dans ses effets de déterritorialisation ${ }^{28}$. Car le comparatisme invite non seulement à sortir l'analyse des cadres nationaux qu'elle se donne trop souvent comme naturels et qui portent avec eux des choix méthodologiques impensés (comme l'invention d'acteurs collectifs très généraux, dispensés de toute incarnation concrète : la Nation, les Français, les «deux peuples» français qui s'affrontent à propos de l'Ancien Régime, « la culture française »), mais aussi à mettre à l'épreuve d'objectivation le lexique même du travail d'objectivation. Soumis à la critique comparatiste, l'Ancien Régime parait ainsi résister aux analyses qui voudraient y voir une réalité historique tangible et incontestable, dotée de propriétés objectives que l'historien n'a qu'à relever, mais aussi à celles qui n'y perçoivent que le produit arbitraire de la performativité du langage révolutionnaire : c'est là la difficulté particulière de l'expression.

28. Voir à ce sujet l'introduction de Barbara Cassin éd., 2004, et Pierre Bourdieu (Actes de la recherche en sciences sociales, 2000 , "Inconscients d'école»), qui voyait en effet dans le comparatisme le moyen «de rendre étrange l'évident par la confrontation avec des manières de penser et d'agir étrangères, qui sont les évidences des autres ». 


\section{Références}

BAECQUE Antoine DE, 1988, La caricature révolutionnaire, Paris, CNRS.

Bely Lucien éd., 1996, Dictionnaire de l'Ancien Régime : royaume de France, XVIeXVIII siècle, Paris, PUF.

BLACK Jeremy, PORTER Roy éd., 1994, Dictionary of Eighteenth Century World History, Oxford, Blackwell.

BÖDEKER Hans-Erich, HINRICHS Ernstéd., 1991, Alteuropa-Ancien Régime-Frühe Neuzeit. Probleme und Methoden der Forschung, Stuttgart, Friedrich Frommann Verlag.

Brunner Otto, Conze Werner, Koselleck Reinhart éd., 1972-1992, Geschichtliche Grundbegriffe. Historisches Lexikon zur politisch-sozialen Sprache in Deutschland, Stuttgart, Ernst Klett Verlag.

CABourdin Guy, VIARD Georges, 1998 (3e éd.), Lexique historique de la France d'Ancien Régime, Paris, Armand Colin.

CASSIN Barbara éd., 2004, Vocabulaire européen des philosophies. Dictionnaire des intraduisibles, Paris, Le Seuil / Le Robert.

ForstmAnn Wilfried, HIRSCHeLmann Ferdinand, 1987, Meyers kleines Lexikon Geschichte, Mannheim, Meyers Lexikonverlag, $376 \mathrm{p}$.

Fuchs Konrad, RAAB Heribert, 1996 (10e éd.), Wörterbuch zur Geschichte, Munich, DeutscherTaschenbuch Verlag.

FURET François, Ozouf Mona, 1988, Dictionnaire critique de la Révolution française, Flammarion, Paris.

Furet François, 1997 (rééd.), «L’Ancien Régime et la Révolution», P. Nora éd., Les lieux de mémoire, Paris, Gallimard (Quarto), p. 2301 et suiv.

Muchembled Robert et al., Dictionnaire de l'Ancien Régime, Paris, Armand Colin, 2004, 298 p.

ReICHARDt Rolf, 2005, "Ancien Régime», R. Asch, F. Jaeger éd., Enzyklopädie der Neuzeit, Stuttgart, J. B. Metzler, t. 1, col. 371-376.

Salvadori Massimo éd., 2000, Enciclopedia Storica, Bologne, Zanichelli.

Tulard Jean, FAYARD Jean-François, Fierro Alfred, 1987, Histoire et dictionnaire de la Révolution française, 1789-1799, Paris, Robert Laffont.

Vallaud Dominique, Dictionnaire historique, Paris, Fayard, 1995.

Venturino Diego, 1988, "La naissance de l'Ancien Régime», Colin Lucas éd., The Political Culture of the French Revolution, Pergamon Press, Oxford, p. 11-40. 